\title{
Fully automated, four-column capillary LC-MS system for maximizing throughput in proteomic analyses
}

\author{
Eric A. Livesay ${ }^{1}$, Keqi Tang ${ }^{1}$, Beverley K. Taylor ${ }^{1}$, Michael A. Buschbach ${ }^{1}$, Derek F. \\ Hopkins ${ }^{1}$, Brian L. LaMarche ${ }^{1}$, Rui Zhao ${ }^{1}$, Yufeng Shen ${ }^{1}$, Daniel J. Orton ${ }^{1}$, Ronald J. \\ Moore $^{1}$, Ryan T. Kelly ${ }^{1}$, Harold R. Udseth ${ }^{1}$, and Richard D. Smith ${ }^{1,{ }^{*}}$ \\ 1 Biological Sciences Division, Environmental Molecular Sciences Laboratory, Pacific Northwest National \\ Laboratory
}

\begin{abstract}
We describe a 4-column, high-pressure capillary liquid chromatography (LC) system for robust, high-throughput LC-MS(/MS) analyses. This system performs multiple LC separations in parallel, but staggers each of them such that the data-rich region of each separation is sampled sequentially. By allowing nearly continuous data acquisition, this design maximizes the use of the mass spectrometer. Each analytical column is connected to a corresponding ESI emitter in order to avoid the use of post-column switching and associated dead volume issues. Encoding translation stages are employed to sequentially position the emitters at the MS inlet. The high reproducibility of this system is demonstrated using consecutive analyses of global tryptic digest of the microbe Shewanella oneidensis.
\end{abstract}

\section{Keywords}

High Throughput proteomics; liquid chromatography; mass spectrometry

\section{INTRODUCTION}

Comprehensive proteomic investigations are challenged by the highly dynamic nature of proteomes and the wide variations in protein molecular weight, isoelectric point (pI), and concentration. In mass spectrometry (MS)-based proteomics, separations are frequently used to overcome dynamic range limitations. The shift over the past decade from offline gel-based separations (i.e., 2D-PAGE) to online separations using capillary liquid chromatography (LC), typically coupled to electrospray ionization ESI-MS(/MS), has resulted in significantly increased throughput. Several innovations, such as the online multidimensional separations pioneered by Yates and coworkers ${ }^{1}$ and the accurate mass and time tag (AMT) method ${ }^{2}$ developed by this group have enabled analyses with coverage comparable to that of 2D-PAGE, but with less sample and in a fraction of the time. ${ }^{3,4}$ For example, Shen et al. reported a 1D LC-MS/MS analysis of global tryptic digest of the microbe Shewanella oneidensis using a linear ion trapmass spectrometer in which $\sim 1,000$ proteins were identified from $2.5 \mu \mathrm{g}$ of sample in 50 min..$^{5}$ However, despite the throughput advantages afforded by capillary LC separations, the low duty cycle of a single-column system indicates that further increases in throughput are possible. The duty cycle of the LC system is defined as the ratio of useful chromatographic separation time to the total time between the beginnings of subsequent

*Correspondence to: Richard D. Smith, Biological Sciences Division, Pacific Northwest National Laboratory, Mail stop: K8-98, Richland, WA, 99354, USA, Phone (509) 376-0723, Fax (509) 376-7722, E-mail: rds@ pnl.gov2007 
chromatographic separations. The low duty cycle of a single-column system stems from the time required to re-equilibrate the column, inject the sample, etc., as well as the dead time at the beginning of a separation particularly with the use of longer columns chosen to provide high peak capacities.

The duty cycle can be significantly improved by operating multiple columns in parallel. Highthroughput, conventional flow rate (e.g., $\sim 1 \mathrm{~mm}$ diameter i.d.), multi-column LC-MS systems developed for the pharmaceutical industry have been used to screen combinatorial libraries and perform pharmacokinetic studies ${ }^{6-18}$. Two parallel techniques - multiplexing and staggered parallel separations - have been pursued for operating non-capillary multi-column LC systems. Multiplexed designs described by de $\operatorname{Biasi}^{8}$ and $\mathrm{Kassel}^{9}$ in 1999 were later commercialized as the MUX interface available from Waters (Milford, MA, USA). The commercial MUX interface consists of either four or eight ESI emitters (one per column) that are aimed at an MS inlet fitted with a rotating aperture used to sequentially sample each emitter. A number of studies and applications using this commercial interface have been reported ${ }^{10-14}$. Staggered parallel separations are distinct from multiplexed separations because the processing of different samples is offset in time. A variety of mechanisms have been employed to accomplish staggered parallel separations ${ }^{15-17}$, and currently, a system that utilizes four fully independent HPLC systems, which are coordinated by proprietary software (developed by Cohesive Technologies; now Thermo Fisher, Franklin, MA, USA) is available as the Aria LX4. ${ }^{18}$

Efforts to improve the throughput of capillary LC systems for proteomics using staggered parallel separations have been pursued in this laboratory by Shen et al., including the development of a dual-column system in which one column was used for analysis while the other was being re-equilibrated 19,20 . In a later implementation, Belov, et al. reported a fully automated capillary LC system operated at 10,000 psi that utilized custom control software and an autosampler that automatically retrieved and injected samples into the fluidic system eliminated the need for a human operator and enabled continuous operation. 21

Herein, we describe an automated 4-column capillary LC system that performs staggered parallel separations and is capable of unattended continuous operation. This system couples two of the aforementioned 2-column systems and thus has two mobile phase sources 20,21 . Since it can alternate between injecting samples on either of its 2-column systems, the automated 4-column system has a duty cycle of nearly $100 \%$ for a broad range of LC conditions. Commercially available encoding translation stages are used to automatically align one column/ESI emitter at a time with the MS inlet for sequential LC-MS analyses ${ }^{22}$. By coupling a unique ESI emitter to each column, dead volume issues associated with coupling all columns to a single emitter using post-column switching are avoided. We report achievable duty cycles for several LC configurations and the demonstrated reproducibility of the system in terms of relative elution times and analyte peak intensities obtained from repeated proteomic analyses.

\section{EXPERIMENTAL}

\section{4-column, 2-mixer system design}

Figure 1 shows a schematic of the 4-column, 2-mixer system. The system uses a total of 11, 2-position valves (VICI Valco, Houston, TX) that are rated to 15,000 psi. The fluidic components of each sub-system consist of a mobile phase mixer and four valves: a 6-port injection valve with a sample loop and three, 4-port valves (one each for mobile phase solvent, column, and purge/split selection). The LC system is operated at constant pressure and the mobile phase exiting the mixer is split before passing through the sample injection valve and the analytical column. Three 10,000 psi syringe pumps (Teledyne ISCO, Lincoln, NE) that share a single controller are used to supply mobile phase to the systems; two of these pumps 
are dedicated to mobile phase A (one for each system) and one is dedicated to mobile phase B. Each pump has a dedicated 2-position, 4-port pump control valve that connects the pump either to the fluidic system (i.e., sub-system 1 or 2) or to a refill reservoir via an on-line degasser (Phenomenex, Torrance, CA; not shown). A single autosampler (LEAP Technologies, Carrboro, NC) with a cooled 3-drawer sample holder (not shown) delivers samples to both systems. Each of the two subsystems utilizes two analytical columns. The four electrospray emitters are connected to a pair of coupled mechanical translation stages that sequentially aligns each emitter with the sampling inlet of the mass spectrometer (described in detail below). A single 1-3 axis universal motion controller (Newport, Irvine, CA) controls both stages.

Software developed in-house to coordinate operation of the system (including sending a trigger to the mass spectrometer to begin data acquisition) runs on a laptop computer that communicates with the various LC system components through a 16-port USB HUB (Digi International, Minnetonka, MN).

The five different LC configurations used to evaluate duty cycle are listed in Table 1 . These configurations are defined by operational pressure, sample loop volume, mixer volume, analytical column characteristics (i.e., the length, internal diameter and size of packing material), flow rates, and length of the gradient elution. The analytical columns consisted of fused-silica capillaries that were packed in-house with porous $3 \mu \mathrm{m}$ or $5 \mu \mathrm{m}$ diameter C18 bonded particles (Phenomenex, Torrance, CA) as described previously 19 . Electrospray emitters were $3-4 \mathrm{~cm}$ long and chemically etched from $20 \mu \mathrm{m}$ i.d. $\times 150 \mu \mathrm{m}$ o.d. fused silica capillaries $^{23}$. The emitters were coupled to the analytical columns using stainless steel unions (VICI Valco, Houston, TX). Mobile phase A was prepared by combining purified water from a Nanopure Infinity system (Barnstead, Dubuque, IA) that had a resistivity $\geq 18 \mathrm{M} \Omega$ with $0.2 \%$ acetic acid (Sigma-Aldrich, St. Louis, MO) and 0.05\% trifluoroacetic acid (Sigma-Aldrich). Mobile phase B consisted of a 90:10 v:v ratio of acetonitrile (Fisher Scientific, Waltham, MA) to nanopure water with $0.1 \%$ trifluoroacetic acid (Sigma-Aldrich).

\section{Positioning electrospray emitters for the multiple-capillary LC system}

The translation stages (Newport, Irvine, CA) used to position electrospray emitters at the mass spectrometer inlet are pictured in Figure S-1a. Each stage is capable of $100 \mathrm{~mm}$ of travel and is coupled to the other stage by a 90-degree angle bracket (Newport). DC actuators that provide $50 \mathrm{~mm}$ of travel and have a bidirectional repeatability of $<2 \mu \mathrm{m}$ are used to position the stages. The horizontal translation stage sits on top of a custom-machined aluminum mounting plate that is used to position the components in front of the mass spectrometer inlet.

The custom-built Delrin ${ }^{\circledR}$ carriage that is used to couple four electrospray emitters onto the vertical translation stage is detailed in Figure S-1b. Stainless steel unions that couple the emitters to the analytical columns fit into slots in the Delrin carriage and are held in place by a set-screw with a spring-loaded ball bearing at one end of the screw. The electrical connection between unions is made through two mechanisms: 1) stainless steel 'bridges' maintain electrical contact between unions that sit side-by-side and 2) metal springs in electrical contact with the screws that hold the metal 'bridges' onto the Delrin carriage maintain the electrical contact between top and bottom electrospray emitters. The ESI voltage source from the mass spectrometer is connected to one of the screws which leads to all of the electrospray emitters being held at the same voltage. At normal operating voltages all of the ESI emitters are electrospraying but only the emitter positioned at the mass spectrometer inlet leads to any detectable signal (i.e., there is no cross-talk between emitters). This is demonstrated by a complete loss of signal upon movement of the stages by $3 \mathrm{~mm}$ (i.e., $17 \%$ of the smallest distance between emitters) while they are held at their normal operating voltage. Electrospray performance for the emitter positioned at the inlet of the mass spectrometer can be affected by sample injection onto another column and is likely due to a temporary drop in the resistance 
along the second column as unretained salts in the sample pass through the column and lead to the current limit of the ESI voltage source being met before its voltage is automatically lowered. An ESI holder that decouples the ESI emitters electrically will address this issue.

While the pumps are pressurized, solvent flows through all 4 emitters continuously, either from the mixer or from a dedicated line for mobile phase. During pump refills, the ESI voltage is set to $0 \mathrm{kV}$.

\section{LC system operation}

The four steps associated with each LC separation- load, gradient elution, purge, regeneration - are depicted in Figure 2a along with the MS data acquisition time. The load step includes the time needed for the autosampler to wash the sample injection syringe, inject a sample onto the sample loop, and load the sample onto the head of the column. The gradient elution step begins when the solvent select valve is switched, which allows mobile phase B to enter the mixer. The purge step consists of flushing the mixer at a relatively high flow rate $(\sim 2 \mathrm{~mL} / \mathrm{min})$ with mobile phase B, followed by a period of time to allow residual bound species to be washed from the column, and then flushing the mixer with mobile phase A. Column regeneration begins as soon as the mixer is fully purged with mobile phase $\mathrm{A}$ and continues using a dedicated line connected to the column select valve (see Figure 1). The gradient elution step can be divided into three regions: dead time, data-rich region, and data-poor region. In the data-poor region, very little data is acquired that is relevant to peptide identifications. The dead time of the gradient elution refers to the delay time required for the solvent gradient to reach the ESI emitter. In our system, the sample loop is bypassed during the gradient elution, purge, and regeneration steps, which minimizes the volume of solvent in the flow path. The ESI emitters are positioned immediately before MS data acquisition begins.

\section{Mass spectrometer}

Data was obtained using an LTQ ion trap mass spectrometer (Thermo Fisher). The ESI voltage was set at $2.2 \mathrm{kV}$ and the heated capillary was maintained at $200^{\circ}$ C.Ions were detected over an $\mathrm{m} / \mathrm{z}$ range of $400-2000$.

\section{Sample preparation}

Sample preparation was similar to that reported previously by Shen et al. ${ }^{24}$ Briefly, $S$. oneidensis was grown chemostatically in-house to steady state prior to harvest. Cell pellets were resuspended and then lysed using $0.1 \mathrm{~mm}$ zirconia/silica beads in a Mini-BeadBeater-8 (BioSpec Products Inc., Bartlesville, OK). The lysate was collected and then subjected to reduction and denaturation with urea, thiourea, and dithiothreitol, after which it was diluted 10-fold with $100 \mathrm{mM} \mathrm{NH}_{4} \mathrm{HCO}_{3}$, $\mathrm{pH}$ 8.4. Following addition of $\mathrm{CaCl}_{2}$, samples were tryptically digested for $3 \mathrm{~h}$ at $37{ }^{\circ} \mathrm{C}$ (1:50 trypsin:protein w:w). Once digested, the samples were cleaned-up using C18 solid phase extraction (SPE) columns (Supelco, St. Louis, MO). Peptides were eluted off of the SPE columns with $4: 1$ ACN: $\mathrm{H}_{2} \mathrm{O}$ that contained $0.1 \%$ TFA, and the resulting sample was concentrated using a Speed Vac (Thermo-Savant, Holbrook, NY) to a final concentration of $26.4 \mu \mathrm{g} / \mu \mathrm{l}$ in $100 \mathrm{uL}$ of solution. This solution was then diluted to a concentration of $0.22 \mu \mathrm{g} / \mu \mathrm{l}$ with $25 \mathrm{mM}$ Ammonium Formate, $\mathrm{pH} 8.0$ and aliquots of 100 $\mu \mathrm{l}$ were stored at $-80^{\circ} \mathrm{C}$.

\section{RESULTS AND DISCUSSION}

\section{Reproducibility}

To evaluate the reproducibility of the 4-column, 2-mixer LC system, 36 consecutive analyses of $500 \mathrm{ng}$ of tryptically digested $S$. onedenisis whole-cell lysate were performed over a period 
of 3.5 days. The system was operated based on configuration 3 (Table 1) and routine conditions were employed that included automated pump refills and a single visual alignment of the ESI emitters prior to the first run. All four columns were used sequentially and were grouped into 9 "cycles" of 4 analyses apiece such that each cycle included one analysis on each column. MS analyses were performed during cycles 1, 3, 5, 7, and 9 and MS/MS analyses during cycles $2,4,6$, and 8 . Reproducibility was evaluated based on the elution times of 10 peaks from across the elution profile that were observed in all $20 \mathrm{MS}$ analyses (Figure 3). Absolute elution times for each LC-MS analysis were determined from inspection of selected ion chromatograms (SIC) for all 10 peaks simultaneously. For comparative purposes, the relative elution times for these peaks were calculated using the method employed by Shen et al., 25 which consisted of linearly normalizing the elution times between 0 and 1 based on the elution times of the earliest and latest eluting peaks chosen for analysis.

All columns of the 4-column, 2-mixer system produced comparable results even for analyses that were performed several days apart. The elution order of the 10 peaks was maintained for all analyses. Variations in elution time are characteristic of variable-flow/constant-pressure LC systems (e.g., single column systems). The 4-column system reported here also exhibited "column-specific" and "sub-system specific" variations. Sub-system-specific variations occur because each pair of columns is physically connected to different sub-systems and are illustrated by similar behavior for columns 1 and 3 and similar behavior for columns 2 and 4 . These variations are significantly reduced by the normalization strategy described above.

Elution time run-to-run reproducibility was evaluated based on the performance of each individual column during cycles 1, 3, 5,7 and 9. The relative peak intensities, absolute elution time of individual peaks, and peak shapes are fairly consistent across all runs. The average relative standard deviation (RSD) values for absolute elution times (10 peaks) for columns 1 , 2,3 and 4 (over all 5 cycles) were $7.5 \%, 1.2 \%, 17.3 \%$, and $0.5 \%$ respectively. The relatively higher run-to-run variations observed for columns 1 and 3 reflect sub-system specific variations. The effects of these variations are significantly reduced by using relative retention times. The average RSD values for relative elution time were $0.7 \%, 0.3 \%, 0.9 \%$ and $0.3 \%$ for columns $1,2,3$, and 4, respectively, which is comparable with published values for the manually-operated 1-column, 1-mixer precursor of the current system. ${ }^{25}$

Column-to-column elution time reproducibility was determined from consecutive analyses using each of the four different columns during a given cycle (e.g., columns 1, 2, 3 and 4 during cycle 1). Normalized chromatograms for the first cycle are shown in Figure 4. Similar to the run-to-run analyses, the average RSD for these column-to-column analyses is the average of the RSD values for each of the 10 peaks observed during a given cycle (i.e., on columns 1, 2, 3 and 4). The average RSD values for absolute elution time were 7.0\%, 15.9\%, 6.5\%, 7.2\%, and $11.6 \%$ for cycles $1,3,5,7$ and 9 , respectively, while the average RSD values for relative elution time were $0.3 \%, 1.1 \%, 0.3 \%, 0.5 \%$, and $0.9 \%$, which are consistent with published results $^{25}$. Like the run-to-run analyses, these variations reflect sub-system specific variations. The effect of subsystem variations on RSD values becomes more apparent when the values for the column that showed the largest deviation from average elution time of the first eluting peak (i.e., $m / z$ 627.83) are excluded from the calculation. For example, the average RSD of absolute elution time dropped to $3.2 \%, 1.2 \%, 2.6 \%, 3.4 \%$, and $1.7 \%$ for cycles $1,3,5,7$, and 9 , respectively, when values for either column 1 or 3 , both on subsystem 1 , were removed from the evaluation.

\section{Duty cycle}

The 4-column, 2-mixer gradient, reversed-phase, capillary LC system is designed to maximize sample throughput, i.e., the number of samples analyzed by the mass spectrometer per unit time, while maintaining comparable chromatographic quality achieved with a 1-column, 1- 
mixer system. Since the mass spectrometer only acquires useful data during the gradient elution step, the highest achievable throughput is obtained when gradient elution steps are performed back-to-back. As shown in Figure 2b, this is not possible with a single-column design. Even with two columns and a single mixer, which allows one column to be regenerated while the other is used for an analysis, there is still wasted time on the mass spectrometer during the load and purge steps between each gradient elution (Figure 2c). Though the time for these steps can be minimized, they cannot be completely removed because the system has only a single gradient mixer, which inherently limits the duty cycle. By using two mixers, analyses can be alternated between a column connected to the first mixer and a column connected to the second mixer (Figures 2d). The four steps can be further staggered such that when the gradient elution ends on one column, the data-rich region of the gradient elution is just beginning on another column (Figure 2e), a technique which we refer to here as elution hopping. Using elution hopping, the LC system operates with a duty cycle of nearly $100 \%$ and the mass spectrometer only samples the data-rich region of each separation.

The theoretical duty cycle for each of the three LC systems (i.e., 1-column, 1-mixer; 2-columns, 1-mixer; 4-columns, 2-mixers) is provided in Table 2 along with relevant time restrictions that must be met for the theoretical and empirical duty cycles to match. The single restriction on a 2-column, 1-mixer system is the result of competition for the single mixer (e.g., the mixer cannot provide mobile phase B for the gradient elution of column 1 while simultaneously supplying mobile phase A for the load step of column 2). A 4-column, 2-mixer system has two restrictions. The first is that the time involved for successive uses of the same column (i.e., purge, regeneration, and load steps) must be less than or equal to the sum of the gradient elution times for the other three columns. The second restriction is that the gradient elution time for a column (e.g., column \#2) connected to mixer \#2 must be greater than or equal to the sum of the purge and load times of the two columns (e.g., columns \#1 and \#3) that share mixer \#1. Note that the restrictions for the 4-column, 2-mixer system without and with elution hopping are related by substituting the gradient elution time with gradient elution time minus dead time and the load time with load time plus dead time.

The benefits of the 4-column, 2-mixer system with elution hopping are illustrated in Figure 5 by comparing its performance to that of a 1-column, 1-mixer system and a 2-column, 1-mixer system for each of the five LC configurations listed in Table 1. In all cases the 4-column, 2mixer system with elution hopping has a higher duty cycle than either of the 1-mixer systems. This benefit is most dramatic for configurations 2, 3 and 4 where the 4-column, 2-mixer system with elution hopping provides more than a $100 \%$ improvement in the duty cycle compared to the 1-column, 1-mixer system. This benefit is reduced for configuration 5 because of the long gradient elution time compared to the times of the other steps; however, the 4-column, 2-mixer system with elution hopping still provides $28 \%$ improvement in duty cycle over the 1 -column, 1-mixer system. Configuration 1 illustrates just the opposite; that is, the gradient elution time is so short compared to the other times that neither of the restrictions listed in Table 2 are satisfied, and the empirical duty cycle of the 4-column, 2-mixer system with elution hopping drops to $6 \%$ compared to $4 \%$ for the 1-column, 1-mixer system. Similarly, but less dramatically, restriction \#2 is not satisfied for the 4-column, 2-mixer system with elution hopping for configuration 2 , which reduces the duty cycle to $91 \%$. We note that to achieve a duty cycle of $\sim 100 \%$ would require the addition of more sub-systems (see Figure 1), e.g., a 6-column, 3mixer system. To maximize the benefit of these additions, pumps dedicated to supplying mobile phase A to each additional sub-system would also have to be added.

Several other aspects that relate to practical operation of the 4-column, 2-mixer system also affect its duty cycle. The first two are that in between each analysis, the ESI tips must be repositioned and the mass spectrometer reset. These processes can be overlapped and are relatively short, taking $\sim 10 \mathrm{~s}$ and $\sim 30 \mathrm{~s}$, respectively. Inclusion of these times in the duty cycle 
calculations for an LC configuration with a gradient elution time of $>\sim 100$ min (i.e., configurations 3, 4 and 5 in Table 2) leads to a duty cycle of $>99 \%$. The third aspect has a much larger effect and is due to automatic pump refills that occur less frequently, but completely stop sample processing while they take place. A pump refill leads to a gap between the end of one gradient elution and the beginning of the next that is equivalent to the time needed for a purge, regeneration, refill ( $18 \mathrm{~min})$, and load and dead times. For example, pump refills for configuration 3 (Table 1) occur after every 10 samples and reduce the overall duty cycle to $87 \%$. Note that the duty cycle between refills remains $>99 \%$. The addition of another pump to this system so that each sub-system would have a dedicated pump for mobile phase A and one for mobile phase B would allow pump refills to be coordinated so that samples could be processed continuously (i.e., only the pumps for one sub-system would be refilled at a time). Alternatively, the loss in duty cycle could be avoided by using different pumps, such as reciprocating piston pumps that can be refilled without interrupting operation.

\section{Extended operation}

Nine fully-automated LC systems in our high-throughput proteomics production facility have been configured as 4-column, 2-mixer systems that operate in elution hopping mode. Operation of these systems, some for more than a year, has demonstrated the significant benefits that this design offers in terms of cost and laboratory space, in addition to data production. For example, a system setup according to configuration 3 in Table 1 was operated for several days as a 4column, 2-mixer system and then as a 1-column system. The practical throughput of the 4column system (e.g., including pump refills) was twice that of the 1-column system - an average of 12 samples vs. 6 samples processed in 24 hours. Furthermore, this increased throughput comes without a need for increased laboratory space. Upgrading an existing 2-column, 1-mixer system to a 4-column, 2-mixer system increases throughput by $\sim 80 \%$ while the cost of this upgrade is significantly less than that of a new LC system and mass spectrometer to provide comparable throughput.

\section{CONCLUSIONS}

Evaluation of our 4-column, 2-mixer high-throughput, capillary LC system designed to perform staggered separations reveals a duty cycle of nearly $100 \%$ when operated within welldefined time parameters for column load, gradient elution, purge, and regeneration steps. The platform alternates between two identical sub-systems to achieve a nearly $100 \%$ duty cycle, a concept that is applicable to a variety of non-identical designs. An important aspect of this system is the use of encoding translation stages for positioning the ESI emitters, i.e., one emitter for each analytical column, at the inlet of the mass spectrometer. By using one emitter per column instead of coupling all 4 columns to a single emitter using a valve avoids degradation of chromatographic quality that would result due to dead volume in the valve. The performance and reproducibility of this system determined from repeated analyses of the microbe $S$. oneidensis is consistent with those obtained for a 1-column, 1-mixer system ${ }^{25}$. Average RSD values of $\sim 1 \%$ for run-to-run and column-to-column relative elution times are observed. Operation of nine 4-column, 2-mixer systems for high throughput LC-MS(/MS) analyses over the past year has demonstrated the robustness of these systems.

\section{Supplementary Material}

Refer to Web version on PubMed Central for supplementary material.

\section{ACKNOWLEDGEMENTS}

We thank Matthew E. Monroe and Navdeep Jaitly for useful discussions regarding data analysis, Heather M. Mottaz for sample preparation and James Ewing for the mechanical drawing shown in Figure S-1b. We thank the U.S. Department of Energy, Office of Biological and Environmental Research for their support of portions of this research. 
Work was performed in the Environmental Molecular Science Laboratory, a U. S. Department of Energy (DOE) national scientific user facility located on the campus of Pacific Northwest National Laboratory (PNNL) in Richland, Washington. PNNL is a multiprogram national laboratory operated for the DOE by Battelle Memorial Institute under contract DE-AC05-76RLO-1830.

\section{REFERENCES}

(1). Link AJ, Eng J, Schieltz DM, Carmack E, Mize GJ, Morris DR, Garvik BM, Yates JR 3rd. Nat Biotechnol 1999;17:676-682. [PubMed: 10404161]

(2). Smith RD, Anderson GA, Lipton MS, Pasa-Tolic L, Shen Y, Conrads TP, Veenstra TD, Udseth HR. Proteomics 2002;2:513-523. [PubMed: 11987125]

(3). Gorg A, Weiss W, Dunn MJ. Proteomics 2004;4:3665-3685. [PubMed: 15543535]

(4). Lopez-Ferrer D, Canas B, Vazquez J, Lodeiro C, Rial-Otero R, Moura I, Capelo JL. Trac-Trends in Analytical Chemistry 2006;25:996-1005.

(5). Shen YF, Smith RD, Unger KK, Kumar D, Lubda D. Analytical Chemistry 2005;77:6692-6701. [PubMed: 16223258]

(6). Tiller PR, Romanyshyn LA, Neue UD. Analytical And Bioanalytical Chemistry 2003;377:788-802. [PubMed: 12904948]

(7). Manisali I, Chen DDY, Schneider BB. Trac-Trends in Analytical Chemistry 2006;25:243-256.

(8). de Biasi V, Haskins N, Organ A, Bateman R, Giles K, Jarvis S. Rapid Communications in Mass Spectrometry 1999;13:1165-1168. [PubMed: 10407292]

(9). Wang T, Cohen J, Kassel DB, Zeng L. Comb Chem High Throughput Screen 1999;2:327-334. [PubMed: 10644858]

(10). Bayliss MK, Little D, Mallett DN, Plumb RS. Rapid Commun Mass Spectrom 2000;14:2039-2045. [PubMed: 11085416]

(11). Yang LY, Mann TD, Little D, Wu N, Clement RP, Rudewicz PJ. Analytical Chemistry 2001;73:1740-1747. [PubMed: 11338587]

(12). Deng Y, Zeng H, Unger SE, Wu JT. Rapid Commun Mass Spectrom 2001;15:1634-1640. [PubMed: 11544604]

(13). Deng Y, Wu JT, Lloyd TL, Chi CL, Olah TV, Unger SE. Rapid Commun Mass Spectrom 2002;16:1116-1123. [PubMed: 11992516]

(14). Fung EN, Chu I, Li C, Liu T, Soares A, Morrison R, Nomeir AA. Rapid Commun Mass Spectrom 2003;17:2147-2152. [PubMed: 12955746]

(15). Cass RT, Villa JS, Karr DE, Schmidt DE Jr. Rapid Commun Mass Spectrom 2001;15:406-412. [PubMed: 11291118]

(16). Xia YQ, Whigan DB, Powell ML, Jemal M. Rapid Commun Mass Spectrom 2000;14:105-111. [PubMed: 10623938]

(17). Van Pelt CK, Corso TN, Schultz GA, Lowes S, Henion J. Anal Chem 2001;73:582-588. [PubMed: 11217766]

(18). King RC, Miller-Stein C, Magiera DJ, Brann J. Rapid Communications in Mass Spectrometry 2002;16:43-52. [PubMed: 11754246]

(19). Shen Y, Zhao R, Belov ME, Conrads TP, Anderson GA, Tang K, Pasa-Tolic L, Veenstra TD, Lipton MS, Udseth HR, Smith RD. Anal Chem 2001;73:1766-1775. [PubMed: 11338590]

(20). Shen Y, Tolic N, Zhao R, Pasa-Tolic L, Li L, Berger SJ, Harkewicz R, Anderson GA, Belov ME, Smith RD. Anal Chem 2001;73:3011-3021. [PubMed: 11467548]

(21). Belov ME, Anderson GA, Wingerd MA, Udseth HR, Tang K, Prior DC, Swanson KR, Buschbach MA, Strittmatter EF, Moore RJ, Smith RD. Journal of the American Society for Mass Spectrometry 2004;15:212-232. [PubMed: 14766289]

(22). Livesay, EA.; Tang, K.; Buschbach, MA.; Shen, Y.; Taylor, BK.; Anderson, GA.; Moore, RJ.; Smith, RD. 53rd ASMS Conference on Mass Spectrometry; San Antonio, TX. 2005; R. Z.poster

(23). Kelly RT, Page JS, Luo QZ, Moore RJ, Orton DJ, Tang KQ, Smith RD. Analytical Chemistry 2006;78:7796-7801. [PubMed: 17105173]

(24). Shen Y, Zhang R, Moore RJ, Kim J, Metz TO, Hixson KK, Zhao R, Livesay EA, Udseth HR, Smith RD. Anal Chem 2005;77:3090-3100. [PubMed: 15889897] 
(25). Shen Y, Zhao R, Berger SJ, Anderson GA, Rodriguez N, Smith RD. Anal Chem 2002;74:42354249. [PubMed: 12199598]

(26). Shen Y, Strittmatter EF, Zhang R, Metz TO, Moore RJ, Li F, Udseth HR, Smith RD, Unger KK, Kumar D, Lubda D. Anal Chem 2005;77:7763-7773. [PubMed: 16316187] 


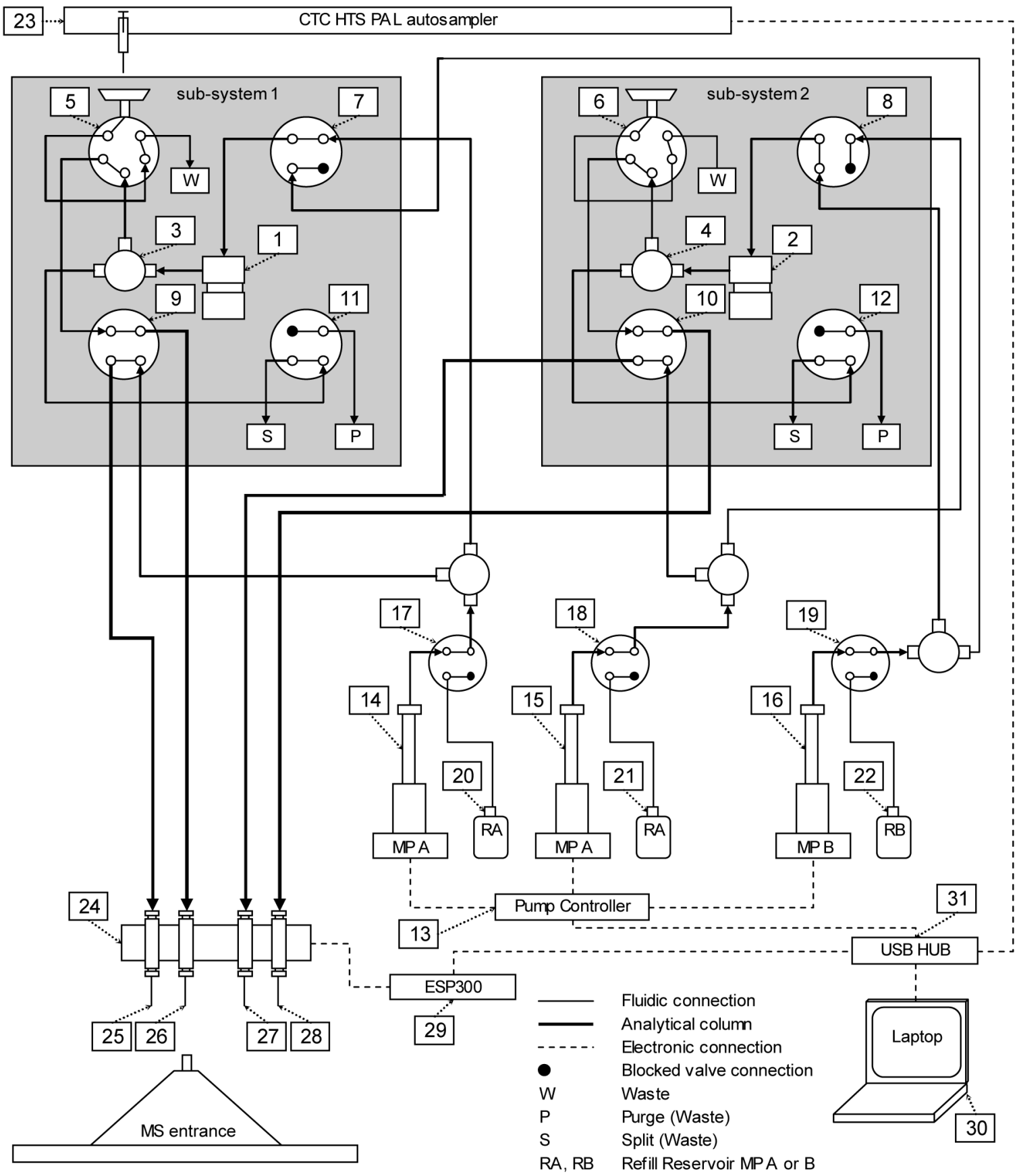

Figure 1.

Schematic of the 4-column, 2-mixer LC system. The components of subsystems 1 and 2 are: $(1,2)$ mixers, $(3,4)$ 'tee' flow split points, $(5,6)$ injection valves, $(7,8)$ mobile phase solvent selection valves, $(9,10)$ column selection valves, $(11,12)$ mixer purge valves. Other components include: (13) syringe pump controller, (14) mobile phase A syringe pump for subsystem 1, (15) mobile phase A syringe pump for subsystem 2 (16) mobile phase B syringe pump for both subsystems, (17) control valve for sub-system 1 pump A, (18) control valve for sub-system 2 pump A, (19) control valve for pump B, (20) refill reservoir for sub-system 1 pump A, (21) refill reservoir for sub-system 2 pump A, (22) refill reservoir for pump B, (23) autosampler that uses a single syringe, (24) encoding translation stages for positioning ESI 
emitters, (25-28) ESI emitters, (29) motion controller for encoding translation stages (30) laptop computer (31) USB HUB. For simplification, several components have not been shown, including electronic connections between the valves and the USB HUB, an on-line degasser and a 3-drawer cool-stack sample holder accessed by the autosampler. The valves of subsystem 1 are shown in positions for sample loading onto the column connected to emitter 26 and the valves of sub-system 2 are shown in positions for a gradient elution on the column connected to emitter 28 . The direction of flow through each connection is illustrated. In the configuration shown there is no flow through the sample loop of sub-system 2 nor through any of the refill reservoirs. 
(a)

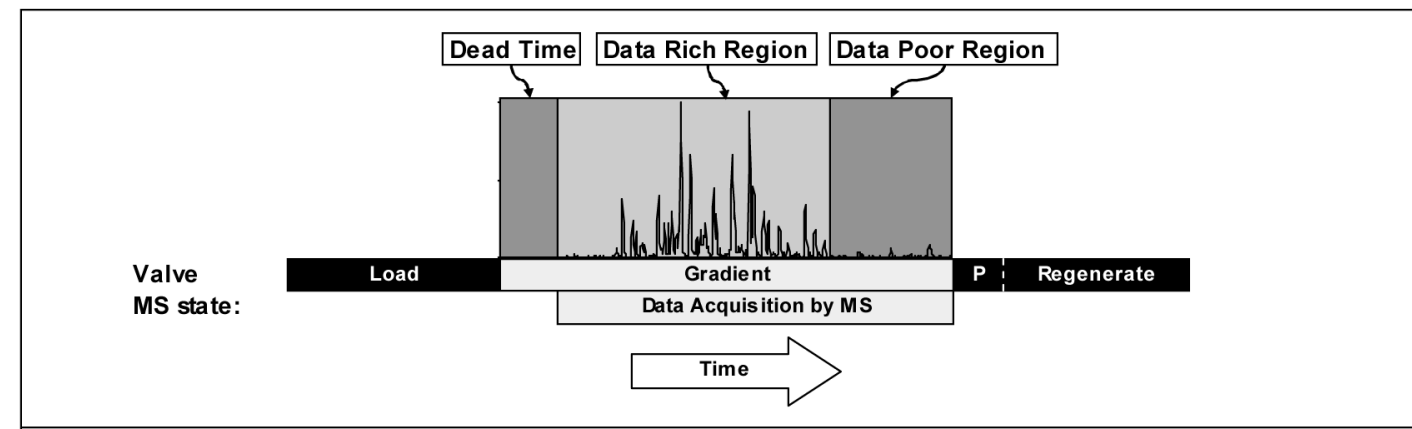

(b)

1-column, 1-mixer

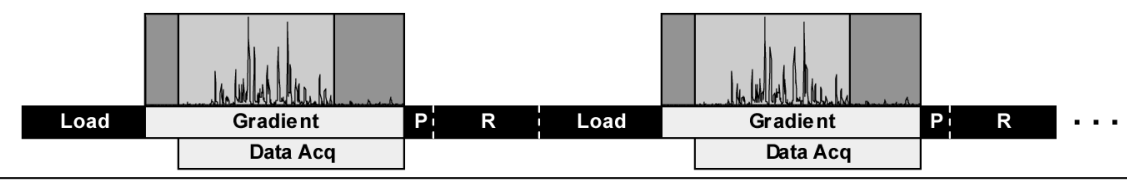

(c)

2-column, 1-mixer

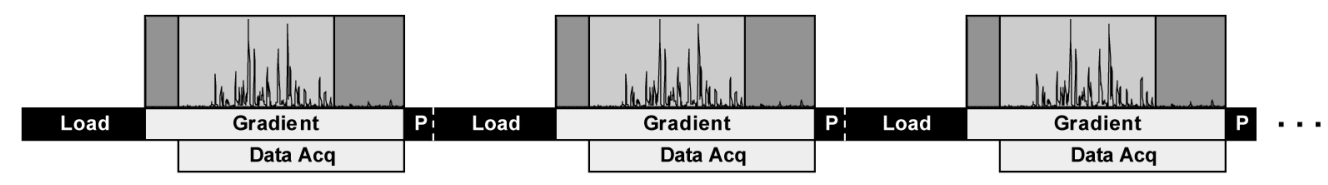

4-column, 2-mixer without elution hopping

(d)

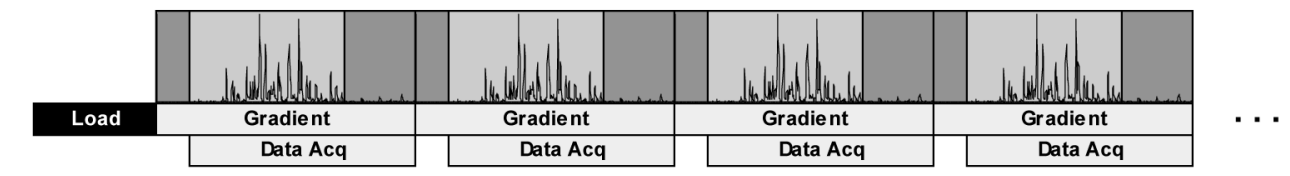

4-column, 2-mixer with elution hopping

(e)

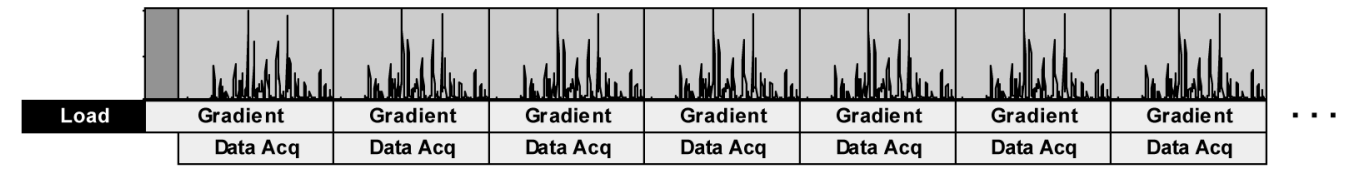

Figure 2.

Duty cycle comparison of various single- and multi-column LC systems with all times drawn to scale for LC configuration 3 in Table 1 except for the data rich region and data poor region which are estimates for the chromatogram which is shown. (a) Schematic description of the steps which must be performed for each sample processed on an analytical column. The steps in consecutive analyses on systems that use (b) 1 column and 1 mixer, (c) 2 columns and 1 mixer, (d) 4 columns and 2 mixers with no elution hopping and (e) the same system shown in (d) but with the dead time at the beginning of the gradient elution bypassed or 'hopped over' and without acquiring data during the 'data poor' region at the end of the gradient elution. 


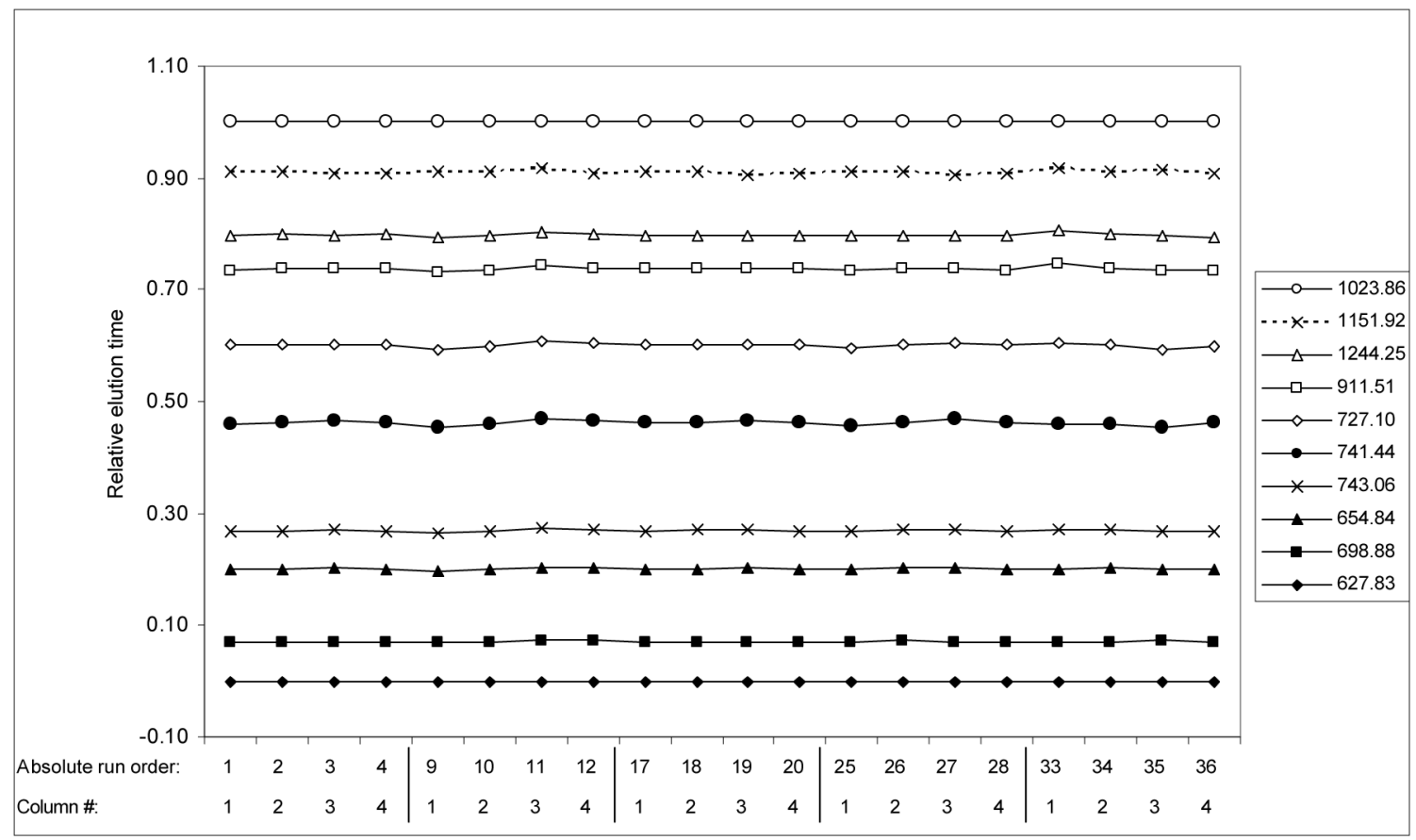

Figure 3.

Comparison of (a) absolute and (b) relative elution times for all 20 MS analyses which are numbered according to the order in which they were run (absolute run order) and the column used for analysis (Column \#). The method for calculating relative elution times is described in the text. The elution times for peaks were obtained by analyzing the selected ion chromatogram for the 10 peaks simultaneously. 


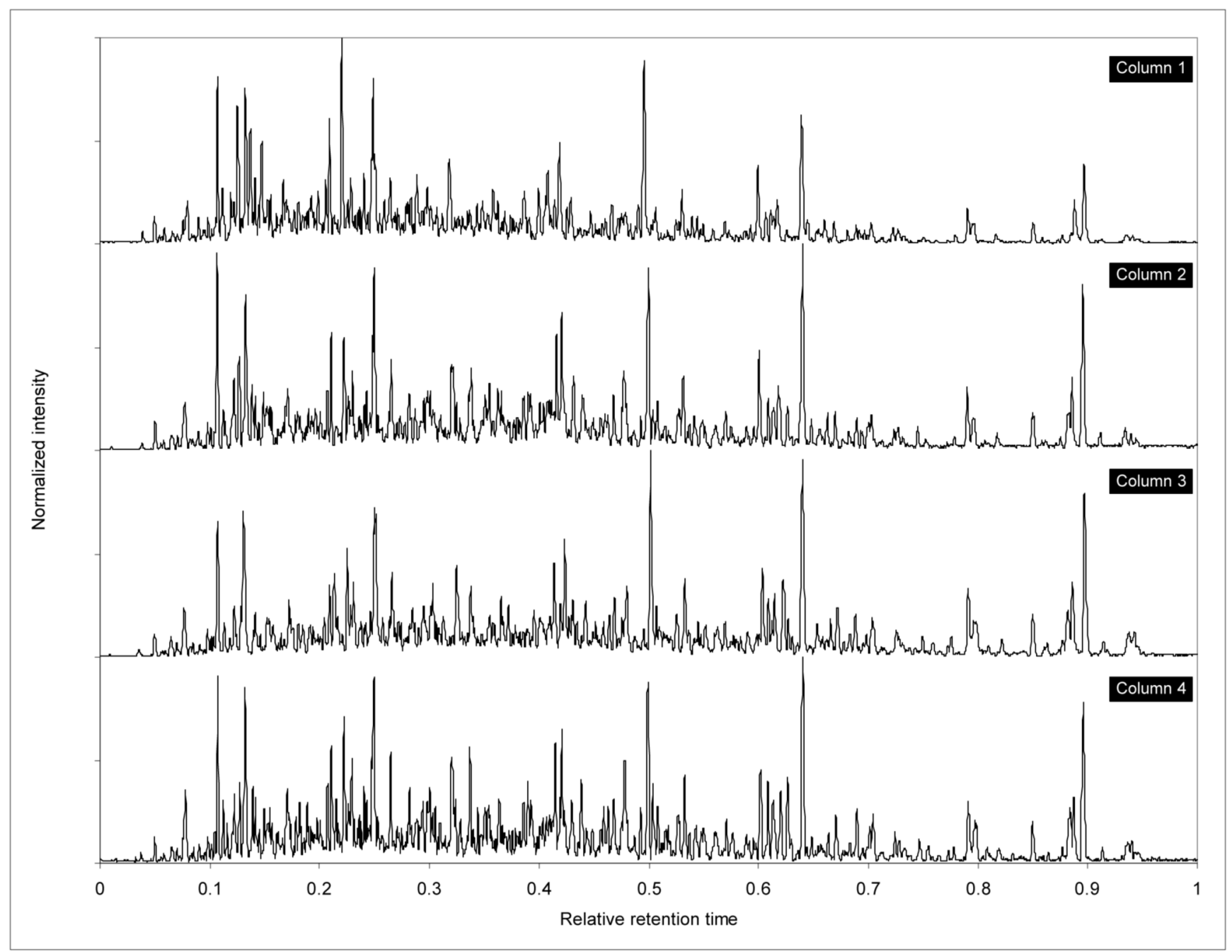

Figure 4.

Comparison between normalized chromatograms for the first cycle of analyses (i.e., consecutive analyses on columns 1,2,3 and 4). Elution times have been linearly normalized based on 2 peaks common to all analyses. Peak intensities have been linearly normalized with the maximum peak intensity set to 1 . 


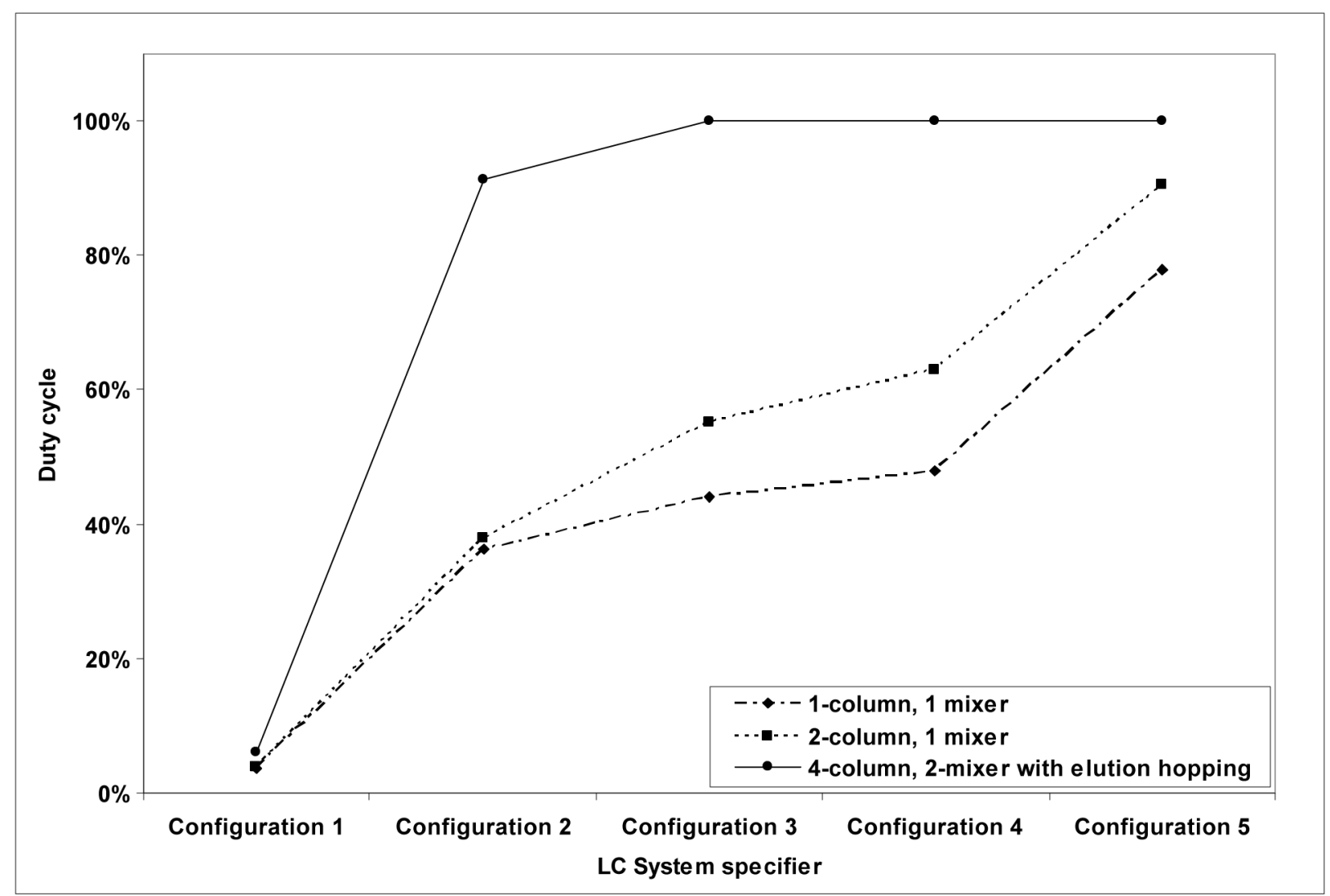

Figure 5.

Duty cycle comparison for all of the LC configurations listed in Table 1. Duty cycles are based on theoretical calculations given in Table 2 and empirical times for chromatographic steps (e.g., load, etc.). 


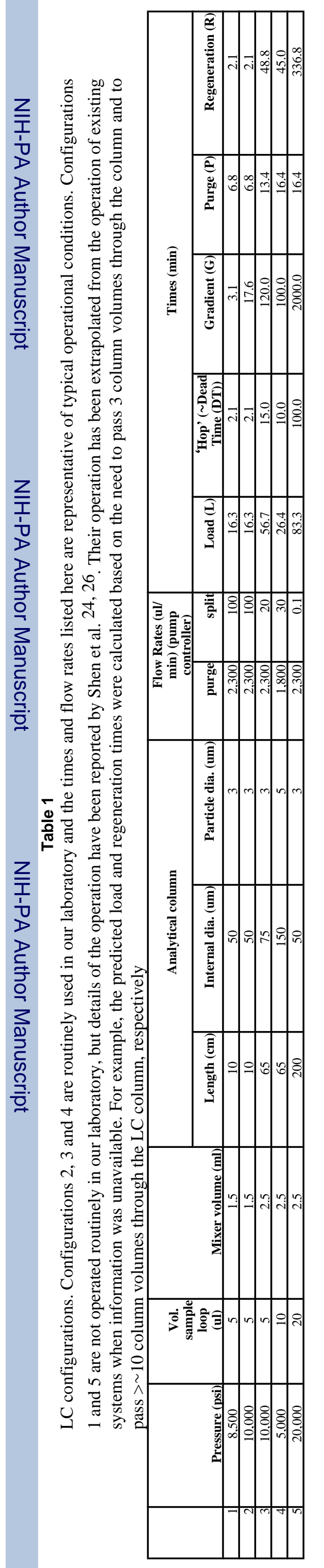


Table 2

Theoretical duty cycle of various LC systems and the restrictions that must be satisfied for the duty cycles to be realized. $D T$ is dead time, and $G, L, P$ and $R_{\max }$ are the times required for gradient elution, sample loading, purging and (maximum) regeneration steps, respectively

\begin{tabular}{|l|c|c|c|}
\hline LC system & Theoretical Dutv Cvcle & Restriction \#1 & Restriction \#2 \\
\hline 1-column, 1-mixer & $\frac{G-D T}{L+G+P+R}$ & N/A \\
\hline 2-column, 1-mixer & $\frac{G-D T}{L+G+P}$ & $L+G+P \geq R_{\max }$ & N/A \\
\hline 4-column, 2-mixer no elution hopping & $\frac{G-D T}{G}$ & $3 G \geq P+R_{\max }+L$ & $G \geq P+L$ \\
\hline 4-column, 2-mixer with elution hopping & $\frac{G-D T-D P}{G-D T-D P}$ & $3(G-D T) \geq P+R_{\max }+L+D T$ & $G-D T \geq P+L+D T$ \\
\hline
\end{tabular}

